\title{
¿PREDICE EL PERFECCIONISMO LAS ALTAS METAS ACADÉMICAS EN ESTUDIANTES CHILENOS DE EDUCACIÓN SUPERIOR?
}

\author{
Nelly G. Lagos San Martín \\ Universidad del Bío-Bío, Chile. \\ Ricardo Sanmartin López \\ Universidad de Alicante, España. \\ María E. Urrea-Solano \\ Universidad de Alicante, España. \\ María J. Hernández-Amorós \\ Universidad de Alicante, España. \\ Lucía Granados Alós \\ Universidad Internacional de Valencia, España. \\ José Manuel García Fernández \\ Universidad de Alicante, España. \\ https://doi.org/10.17060/ijodaep.2017.n1.v2.943
}

Fecha de Recepción: 2 Marzo 2017

Fecha de Admisión: 1 Abril 2017

\section{RESUMEN}

Los trabajos científicos muestran la importancia de contar con altas metas académicas para el logro de un buen rendimiento escolar de los estudiantes de todos los niveles educativos, motivo por el cual resulta necesario delimitar dichas metas, definiendo las variables que las determinan. Es por ello que el objetivo del estudio fue analizar la capacidad predictiva del perfeccionismo sobre las metas académicas en una muestra de 479 estudiantes chilenos de educación superior, 237 hombres y 242 mujeres, sus edades se ubican en un rango que abarca entre los 19 y 36 años de edad. El perfeccionismo se evaluó con la Child and Adolescent Perfectionism Scale (CAPS) y las metas académicas con el Cuestionario de Metas Académicas (CEMA). Los datos fueron analizados a partir del método de regresión logística los cuales permiten informar que altas puntuaciones en perfeccionismo auto-orientado a críticas predicen las altas metas en refuerzo social y que altas puntuaciones en perfeccionamiento auto-orientado al esfuerzo predicen las altas metas de aprendizaje y de logro. Con estos resultados es posible indicar que efectivamente el perfeccionismo predice las altas metas académicas y que esta relación da cuenta de la forma en que se configuran las motivaciones de los estudiantes en el contexto universitario.

Palabras claves: Metas de aprendizaje, Perfeccionismo, Educación superior, Regresión logística 


\section{¿PREDICE El PERFECCIONISMO LAS ALTAS METAS ACADÉMICAS EN ESTUDIANTES CHILENOS DE EDUCACIÓN SUPERIOR?}

\section{ANTECEDENTES}

Las metas académicas revisten de una importancia mayor en el nivel universitario debido a que en este nivel educativo los estudiantes son más autónomos en la adquisición de conocimientos y habilidades y porque el aspecto disposicional también tiende a ser más significativo (Daura, 2015). De ahí que establecer cuáles son las metas o motivaciones que orientan el aprendizaje de los estudiantes universitarios, así como los factores que pueden influir en éstas, sean temáticas de un creciente interés científico y de una importancia trascendental de entrada y permanencia en la universidad (Durán-Aponte y Arias-Gómez, 2015).

Las metas académicas son definidas como los resultados que se desean alcanzar y que orientan el quehacer de los estudiantes (Pool-Cibrian y Martínez-Guerrero, 2013). Por lo que las acciones pueden ser consideradas orientaciones que se direccionan en función de lo que se pretenda obtener buenos resultados, aprender o ser reconocido por otros- (Hayamizu y Weiner, 1991).

Diversos estudios plantean que las metas de aprendizaje que dirigen las acciones de los estudiantes universitarios, se relacionan con el uso de estrategias de aprendizaje específicas, indicándose que altas metas de aprendizaje se asocian con una mayor utilización de estrategias de procesamiento profundo y que bajas metas de aprendizaje se relacionan con una menor utilización de estas estrategias cognitivas y de autorregulación (Braten y Olaussem, 2005; Radosevich, Vaidyanathan, Yeo, y Radosevich, 2004). También se ha encontrado un patrón cognitivo en el que se relacionan las metas de aprendizaje, las atribuciones causales adecuadas (Díaz, Pérez, GonzalézPienda, Núñez, y Rosário, 2008) aseverándose que las metas de aprendizaje se vincularían a atribuciones causales controlables e inestables y que las metas orientadas a resultados se asociarían con atribuciones fijas y estables (Dweck, 1986). Ello porque a juicio de los autores, las atribuciones a causas internas modificables (esfuerzo) o estables y positivas (autoimagen positiva) constituyen aspectos favorables que determinan la motivación por aprender de los estudiantes universitarios (Díaz, Pérez, González-Pienda, Núñez y Solar, 2004; Díaz et al., 2008).

El interés por este tipo de estudios, se debe principalmente al carácter multidimensional de las metas académicas que permite relacionarlo con otros factores de relevancia científica, así como también por su importante valor motivacional (Esteves, Rodríguez, Valle, Requeiro y Piñeiro, 2016; Rodríguez, Cabanach, Piñeiro, Valle, Núñez y González-Pienda, 2001). Motivos por los cuales, en este estudio se ha determinado oportuno relacionarlo con un constructo de interés creciente y que puede influenciar el tipo de metas académicas de los estudiantes universitarios, estableciendo si el perfeccionismo predice las metas académicas.

En psicología, el perfeccionismo es entendido como un rasgo de personalidad que implica para un sujeto el planteamiento de metas altamente difíciles de cumplir, -por sí mismo o por los demás-, impresión que además se acompaña de altas exigencias por el desempeño propio, una valoración autocrítica de la conducta, una excesiva preocupación por cometer errores y el convencimiento de que los demás esperan de él resultados extraordinarios (Hewitt y Flett 1991, citado en Aguilar, Colmenares y Barroeta, 2014). Desde el aspecto emocional se ha señalado que los perfeccionistas tienden a evitar emociones que les recuerden que han fracasado, pero también minimizan sus logros con lo que la emoción asociada también se reduce (Keegan, 2016).

El perfeccionismo es un constructo que al igual que las metas académicas, se ha relacionado con otras variables como los rasgos de personalidad, el afecto 0 la agresividad (Vicent, 2017). También existen estudios que han relacionado este constructo con el rendimiento académico, informando hallazgos discrepantes respecto de esta relación.

Por una parte, algunas investigaciones revelan que los estudiantes no perfeccionistas tienden a obtener promedios académicos menores que los perfeccionistas, refiriéndose tanto a estudiantes 
con un perfil adaptativo como a aquellos con un perfil desadaptativo (Grzegorek, Slaney, Franze y Rice, 2004; Rice y Slaney, 2002; Rice y Ashby, 2007). Por otra, se ha informado que el perfeccionismo desadaptativo podría acarrear un impacto negativo en el rendimiento académico, asociándose con diversos trastornos y contribuyendo al desgaste psicológico e incluso al abandono o postergación de metas (Véase Guido et al, 2014 para una revisión). Desde esta perspectiva, algunos autores (Soria, Martínez, Bresó, Llorens y Grau, 2005) identificaron elementos que en los estudiantes operarían como obstáculos o como facilitadores en el cumplimiento de tareas académicas.

\section{OBJETIVOS}

Analizar la capacidad predictiva del perfeccionismo sobre las metas de aprendizaje en estudiantes chilenos de educación superior.

\section{PARTICIPANTES}

En este estudio participaron 479 estudiantes chilenos, 237 hombres y 242 mujeres, $49.47 \%$ y $50.53 \%$ respectivamente, con edades comprendidas entre 19 y 36 años $(M=22,10$ y $D E=1,34)$, todos ellos estudiantes de $1^{\circ}, 2^{\circ}$ y $3^{\circ}$ año de 6 carreras de Pedagogía de la Universidad del Bío-Bío, sede Chillán (Pedagogía en Historia y Geografía, Pedagogía en Castellano y Comunicación, Pedagogía en Inglés, Pedagogía en Educación Física, Pedagogía en Educación General Básica y Pedagogía en Educación Parvularia).

\section{MÉTODO}

\section{Instrumentos}

En este estudio se utilizaron dos instrumentos, uno para medir el perfeccionismo (CAPS) y el otro para medir las metas académicas (CEMA)

Child and Adolescent Perfectionism Scale (CAPS; Flett, Hewitt, Boucher, Davidson y Munro, 2000). En este estudio se utilizó la versión validada en España (Vicent-Juan, 2017). Este Instrumento evalúa el perfeccionismo en la niñez y la juventud, tiene 22 ítems distribuidos en 3 factores, perfeccionismo socialmente prescrito (PSP), perfeccionismo auto-orientado-críticas (PAO-C) y perfeccionamiento auto-orientado-esfuerzo (PAO-E). Los ítems de este instrumento se responden a través de una escala tipo likert de 5 puntos, reflejando mayor perfeccionismo las puntuaciones más altas. Los índices de confiabilidad de la CAPS para este estudio para cada factor fue de $\alpha=.88$ para (PSP), de $\alpha=.87$ para (PAO-C) y de $\alpha=.90$ para (PAO-E).

El primer factor (PSP) da cuenta de una faceta interpersonal, que implica la representación de las exigencias externas al sujeto a través de la imposición de expectativas de desempeño muy altas y la tendencia a juzgar duramente los resultados" (Vicent-Juan, Inglés, Gonzálvez, Sanmartín y García-Fernández, 2016), las el segundo factor PA0-C mide la autocrítica y representa los aspectos desadaptativos del PAO, finalmente el factor PAO-E evalúa los esfuerzos por la perfección y representa los aspectos adaptativos del PAO.

Cuestionario de Metas Académicas (CEMA; Hayamizu y Weiner, 1991) En este estudio se utilizó la versión española de Núñez, González-Pienda, González-Pumariega, García y Roces (1997). Este instrumento evalúa las metas académicas considerando tres de metas: de aprendizaje, de logro y de refuerzo social. Se compone de 20 ítems que son respondidos en una escala likert de 0 a 4 puntos. La confiabilidad del instrumento para este estudio por escala fue de $\alpha=.86$ para la escala de metas de aprendizaje, de $\alpha=.80$ para metas de logro y de $\alpha=.83$ para metas de refuerzo social.

Las Metas de aprendizaje miden la tendencia de los estudiantes hacia la búsqueda del incremento de su propia competencia, mediante la adquisición y dominio de nuevas habilidades y cono- 


\section{¿PREDICE El PERFECCIONISMO LAS ALTAS METAS ACADÉMICAS EN ESTUDIANTES CHILENOS DE EDUCACIÓN SUPERIOR?}

cimientos y perfeccionando su ejecución en las tareas de aprendizaje, las metas de refuerzo social miden la tendencia de los estudiantes a aprender con el propósito de obtener aprobación y evitar rechazo por parte de los profesores y padres y las metas de logro evalúan la tendencia de los estudiantes a aprender con el propósito de obtener buenos resultados en las evaluaciones.

\section{Procedimiento}

En primer lugar se seleccionaron las carreras en las cuales se llevaría a cabo el estudio, luego se realizó una reunión con los directores de dichas carreras para solicitar los permisos pertinentes y acordar los horarios más propicios para la aplicación de instrumentos. Seguido, se pidió la colaboración de los estudiantes a partir de la firma de los consentimientos informados y se realizó la administración de los cuestionarios en el aula y en el horario convenido.

\section{Análisis de datos}

Los datos fueron examinados a partir de análisis de regresión logística binaria siguiendo el método de regresión por pasos hacia adelante basado en el estadístico de Wald, modelo que permite estimar la probabilidad de que un evento, suceso o resultado ocurra en función de la presencia de uno 0 más predictores.

La capacidad predictiva es estimada a partir del estadístico OR (odd ratio) el cual es interpretado, siguiendo a De Maris (2003), del siguiente modo; si el valor $O R>1$, la predicción es positiva, si $\mathrm{OR}<1$ la predicción es negativa y si $\mathrm{OR}=1$ esto indica que no hay predicción.

Para realizar los análisis de este estudio se dicotomizó la variable criterio (metas académicas) considerando dos grupos; uno que incluye las puntuaciones iguales o inferiores al centil 25 definido como bajas metas académicas y otro con las puntuaciones iguales o superiores al centil 75 definido como altas metas académicas. Las variables predictoras, -desprendidas del cuestionario de perfeccionismo- se consideraron continuas.

Todos los análisis fueron realizados con el programa informático SPSS 20.

\section{RESULTADOS}

Los datos permitieron crear tres modelos de regresión logística a partir de los cuales se puede indicar que en primer lugar, altas puntuaciones en perfeccionismo auto-orientado-críticas predice las altas metas en refuerzo social, en segundo lugar que altas puntuaciones en perfeccionamiento auto-orientado al esfuerzo predice las altas metas de aprendizaje y de logro.

El primer modelo permite hacer una estimación correcta para un $59.7 \%$ de los casos $\left(\chi^{2}=\right.$ $22.82 ; p<.000)$. Los componentes del modelo expresados por la odd ratio $(\mathrm{OR})$ indican que la probabilidad de presentar altas metas de refuerzo social es mayor, concretamente 1.24 por cada punto de aumento en perfeccionismo auto-orientado-críticas (véase tabla 1).

Tabla 1: Regresión logística binaria para la probabilidad de presentar altas metas de refuerzo social en función del perfeccionismo auto-orientado-críticas.

\begin{tabular}{|c|c|c|c|c|c|c|c|c|c|}
\hline Variable & & $\chi^{2}$ & $\mathrm{R}^{2}$ & B & E.T. & Wald & $p$ & OR & I.C. $95 \%$ \\
\hline & Clasificados & & & & & & & & \\
\hline & correctamente & 22.82 & .21 & & & & & & \\
\hline & $59.7 \%$ & & & & & & & & \\
\hline \multirow[t]{2}{*}{ MRS } & & & & .23 & .05 & 18.83 & 000 & 1.26 & $1.13-1.40$ \\
\hline & Constante & & & -1.48 & .39 & 14.42 & 000 & .22 & \\
\hline
\end{tabular}

Nota $:$ MRS $=$ Metas de Refuerzo Social; $\chi^{2}=$ Chi cuadrado; $\mathrm{R}^{2}=$ Cuadrado de Nagelkerke; $\mathrm{B}=$ Coeficiente de regresión; E.T.= Error estándar; Wald $=$ Prueba de Wald; $p=$ Probabilidad; OR = Odd ratio; I.C. = Intervalo de confianza al $95 \%$. 
El segundo modelo permite hacer una estimación correcta para un $61.9 \%$ de los casos $\left(\chi^{2}=\right.$ $16.76 ; p<.000)$. Los componentes del modelo expresados por la odd ratio $(\mathrm{OR})$ indican que la probabilidad de presentar altas metas de aprendizaje es mayor, concretamente 1.23 por cada punto de aumento en perfeccionismo auto-orientado-esfuerzo (véase tabla 2).

Tabla 2: Regresión logística binaria para la probabilidad de presentar altas metas de aprendizaje en función del perfeccionismo auto-orientado-esfuerzo.

\begin{tabular}{|c|c|c|c|c|c|c|c|c|c|}
\hline Variable & & $\chi^{2}$ & $\mathrm{R}^{2}$ & B & E.T. & Wald & $p$ & OR & I.C. $95 \%$ \\
\hline & Clasificados & & & & & & & & \\
\hline & correctamente & 16.76 & .14 & & & & & & \\
\hline & $61.9 \%$ & & & & & & & & \\
\hline \multirow[t]{2}{*}{ MA } & & & & .20 & .05 & 14.36 & 000 & 1.23 & $1.10-1.37$ \\
\hline & Constante & & & -1.94 & .59 & 10.56 & 001 & .14 & \\
\hline
\end{tabular}

Nota: $\mathrm{MA}=$ Metas de Aprendizaje; $\chi^{2}=$ Chi cuadrado; $\mathrm{R}^{2}=$ Cuadrado de Nagelkerke; $\mathrm{B}=$ Coeficiente de regresión; E.T. $=$ Error estándar; Wald $=$ Prueba de Wald; $p=$ Probabilidad; OR $=$ Odd ratio; I.C. $=$ Intervalo de confianza al $95 \%$.

El tercer modelo permite hacer una estimación correcta para un $63.3 \%$ de los casos $\left(\chi^{2}=18.62\right.$; $p<.000)$. Los componentes del modelo expresados por la odd ratio $(\mathrm{OR})$ indican que la probabilidad de presentar altas metas de logro es mayor, concretamente 1.25 por cada punto de aumento en perfeccionismo auto-orientado-esfuerzo (véase tabla 3).

Tabla 3: Regresión logística binaria para la probabilidad de presentar altas metas de logro en función del perfeccionismo auto-orientado-esfuerzo.

\begin{tabular}{|c|c|c|c|c|c|c|c|c|c|}
\hline Variable & & $\chi^{2}$ & $\mathrm{R}^{2}$ & B & E.T. & Wald & $p$ & OR & I.C. $95 \%$ \\
\hline & Clasificados & & & & & & & & \\
\hline & correctamente & 18.62 & .14 & & & & & & \\
\hline & $63.3 \%$ & & & & & & & & \\
\hline \multirow[t]{2}{*}{ ML } & & & & .22 & .05 & 15.96 & 000 & 1.25 & $1.24-1.40$ \\
\hline & Constante & & & -2.27 & .62 & 13.23 & 000 & .10 & \\
\hline
\end{tabular}

Nota: $\mathrm{ML}=$ Metas de Logro; $\chi^{2}=$ Chi cuadrado; $\mathrm{R}^{2}=$ Cuadrado de Nagelkerke; $\mathrm{B}=\mathrm{Coeficiente}$ de regresión; E.T.= Error estándar; Wald $=$ Prueba de Wald; $p=$ Probabilidad; OR $=$ Odd ratio; I.C. $=$ Intervalo de confianza al $95 \%$.

\section{CONCLUSIONES}

Las metas académicas corresponden a las motivaciones que conducen a los estudiantes a realizar acciones para el logro de dichos propósitos proyectados. Por lo que la comprensión del funcionamiento de las metas constituye un aspecto de mucha importancia para avanzar en la comprensión de los procesos involucrados en el desempeño y el aprendizaje de los estudiantes a fin de planear prácticas educativas que permitan promoverlos, principalmente en el nivel educativo superior (Gaeta, Cavazos, Sánchez, Rosário y Högemann, 2015).

Para dar cuenta de la motivación por el estudio, Hayamizu y Weiner (1991) plantearon la presencia de tres tipos de orientaciones o metas; de aprendizaje, de refuerzo social y de logro, los cuales han sido corroborados por los variados estudios que se han generado a partir de este referente teórico.

Las metas académicas se han relacionado con otros constructos de gran relevancia científica, 


\section{¿PREDICE El PERFECCIONISMO LAS ALTAS METAS ACAdÉMICAS EN ESTUDIANTES CHILENOS DE EDUCACIÓN SUPERIOR?}

asimismo en este estudio se comprobó que es predicha por el perfeccionismo. Con lo cual se corrobora la hipótesis inicial, no pudiendo señalar si este resultado es coherente con estudios anteriores debido a que no se han encontrado otras investigaciones que las hayan vinculado con el perfeccionismo en el nivel educativo superior.

Finalmente, señalar que los hallazgos encontrados pueden considerarse muy coherentes con la teoría de base, ya que se encontró que son justamente los estudiantes con altas puntuaciones en perfeccionismo autorientado a críticas quienes presentarían metas altas en refuerzo social y los estudiantes con altas puntuaciones en perfeccionismo autorientado al esfuerzo quienes presentarían altas metas de logro y de aprendizaje.

\section{BIBLIOGRAFÍA}

Aguilar, L., Colmenares, D. y Barroeta, G. (2014). Consideraciones respecto a las normas de una medida de perfeccionismo académico para estudiantes universitarios. Revista de Estudios Clínicos e Investigación Psicológica, 4(7), 4-13.

Braten, I. y Olaussen, B. (2005). Profiling individual differences in student motivation: A longitudinal cluster-analytic study in different academic contexts. Contemporary Educational Psychology, 30, 359-396. doi.org/10.1016/j.cedpsych.2005.01.003

Daura, F. T. (2015). Aprendizaje autorregulado y rendimiento académico en estudiantes del ciclo clínico de la carrera de Medicina. Revista electrónica de investigación educativa, 17(3), 28-45. Recuperado en 04 de febrero de 2017, de http://www.scielo.org.mx/scielo.php?script=sci_arttext\&pid=S1607-40412015000300003\&lng=es\&tlng=es.

Díaz, A., Pérez, V., Gonzaléz-Pienda, J. A., Núñez, C. y Rosário, P. (2008). Patrones atribucionales, metas de estudio, autoconcepto académico y desempeño en estudiantes universitarios. Paper presented at the meeting of the Actas VIII Jornadas Internacionales de Psicología Educacional, Lima, Perú.

Díaz, A., Pérez, V., González-Pienda, J. A., Núñez, C. y Solar, M. (2004). Atribuciones causales y autoconcepto académico en estudiantes universitarios. Paideia, 36, 77-93

Durán-Aponte, E. y Arias-Gómez, D. (2015). Orientación a las metas académicas, persistencia y rendimiento en estudiantes del Ciclo de Iniciación Universitaria. Revista de Docencia Universitaria, 13(2), 189-205. doi: http://dx.org/10.4995/redu.2015.5444

Dweck, C. (1986). Motivational process affecting learning. American Psychologist, 41(10), 10401048. doi: 10.1037/0003-066X.41.10.1040

Esteves, I., Rodríguez, S., Valle, A., Requeiro, B. y Piñeiro, I. (2016). Incidencia de las metas académicas del alumnado de secundaria en su gestión motivacional. Aula Abierta, 44(2), 83-90. doi: 10.1016/j.aula.2016.03.001

Flett, G. L., Hewitt, P. L., Boucher, D. J., Davidson, L. A. y Munro, Y. (2000). The Child Adolescent Perfectionism Scale: development, validation, and association with adjustment. Manuscrito inédito, York University, Toronto, Ontario, Canadá.

Gaeta, M., Cavazos, J., Sánchez, A., Rosário, P. y Högemann, J. (2015). Propiedades psicométricas de la versión mexicana del la Evaluación de Metas Académicas (CEMA). Revista Latinoamericana de Psicología, 47(1), 16-24. doi: 10.1016/S0120-0534(15)30002-9

Grzegorek, J. L., Slaney, R. B., Franze, S. y Rice, K. G. (2004). Self-criticism, dependency, selfesteem, and grade point average satisfaction among clusters of perfectionists and nonperfectionists. Journal of Counseling Psychology, 51(2), 192-200. doi: 10.1037/0022-0167.51.2.192

Guido, F., Galarregui, M., Miracco, M., Partarrieu. A., De Rosa, L., Lago, A... y Traiber, L. (2014). Perfeccionismo y desempeño académico en estudiantes universitarios de la ciudad autónoma 
de buenos aires. Acta Colombiana de Psicología, 17(1), 71-77. doi: 10.14718/ACP.2014.17.1.8 Hayamizu, T. y Weiner, B. (1991). A test of Dweck's model of achievement goals as related to perceptions of ability. The Journal of Experimental Education, 59(3), 226-234. doi: 10.1080/00220973.1991.10806562

Keegan, E. (2016). Evaluación de una Intervención Psicoeducativa sobre Perfeccionismo en Estudiantes Universitarios Intersecciones. Revista Electrónica de la Facultad de Psicología de la $U B A, 6(21)$.

Radosevich, D., Vaidyanathan, V., Yeo, S. y Radosevich, D. (2004). Relating goal orientation to selfregulating processes: A longitudinal field test. Contemporary Educational Psychology, 29(3), 207-229. doi: 10.1016/S0361-476X(03)00032-8

Rodríguez, S., Cabanach, R., Piñeiro, I., Valle, A., Núñez, C. y González-Pienda, J. (2001). Metas de aproximación, metas de evitación y múltiples metas académicas. Psicothema, 13(4), 546-550.

Soria, M., Martínez, I., Bresó, E., Llorens, S. y Grau, R. (2005). Bienestar psicológico en estudiantes universitarios: facilitadores y obstaculizadores del desempeño académico. Anales de Psicología de la Universidad de Murcia, 21(1), 170-180.

Núñez, J., González-Pienda, J., González-Pumariega, S, García, M. y Roces, C. (1997). Cuestionario para la Evaluación de Metas Académicas en Secundaria (CEMA-II). Oviedo, España: Departamento de Psicología, Universidad de Oviedo.

Pool-Cibrian, W. J. y Martínez-Guerrero J. I. (2013). Autoeficacia y uso de estrategias para el aprendizaje autorregulado en estudiantes universitarios. Revista Electrónica de Investigación Educativa, 15(3), 21-37. Recuperado de http://redie.uabc.mx/vol15no3/contenido-poolmtnez.html

Rice, K. G. y Ashby, J. S. (2007). An efficient method for classifying perfectionists. Journal of Counseling Psychology, 54(1), 72-85. doi: 10.1037/0022-0167.54.1.72

Rice, K. y Slaney, R. B. (2002). Clusters of Perfectionistics: Two Studies of Emotional Adjustment and Academic Achievement. Measurement and Evaluation in Counseling and Development, 31(1), 35-48.

Vicent-Juan, M. (2017). Estudio del perfeccionismo y su relación con variables psicoeducativas en la infancia tardía. (Tesis doctoral inédita). Universidad de Alicante, Alicante.

Vicent-Juan, M., Inglés, C., Gonzálvez, C., Sanmartín, R. y García-Fernández, J.M. (2016). Perfeccionismo socialmente prescrito y los cinco grandes rasgos de la personalidad en niños españoles. European Journal of Investigation in Health, Psychology and Education, 6(2), 107118. doi: 10.1989/ejihpe.v6i2.162 
